\title{
Probing Balitsky-Fadin-Kuraev-Lipatov Dynamics in the Dijet Cross Section at Large Rapidity Intervals in $p \bar{p}$ Collisions at $\sqrt{s}=1800$ and $630 \mathrm{GeV}$
}

B. Abbott,${ }^{46}$ M. Abolins, ${ }^{43}$ V. Abramov,${ }^{19}$ B. S. Acharya, ${ }^{13}$ D. L. Adams,${ }^{53}$ M. Adams,${ }^{30}$ V. Akimov, ${ }^{17}$ G. A. Alves, ${ }^{2}$ N. Amos, ${ }^{42}$ E. W. Anderson, ${ }^{35}$ M. M. Baarmand ${ }^{48}$ V. V. Babintsev, ${ }^{19}$ L. Babukhadia,${ }^{48}$ A. Baden,${ }^{39}$ B. Baldin,${ }^{29}$

S. Banerjee, ${ }^{13}$ J. Bantly, ${ }^{52}$ E. Barberis,${ }^{22}$ P. Baringer, ${ }^{36}$ J. F. Bartlett, ${ }^{29}$ U. Bassler, ${ }^{9}$ A. Bean, ${ }^{36}$ A. Belyaev,,${ }^{18}$ S. B. Beri, ${ }^{11}$ G. Bernardi, ${ }^{9}$ I. Bertram,${ }^{20}$ V. A. Bezzubov, ${ }^{19}$ P.C. Bhat,${ }^{29}$ V. Bhatnagar, ${ }^{11}$ M. Bhattacharjee,${ }^{48}$ G. Blazey, ${ }^{31}$ S. Blessing, ${ }^{27}$ A. Boehnlein, ${ }^{29}$ N. I. Bojko, ${ }^{19}$ F. Borcherding, ${ }^{29}$ A. Brandt,${ }^{53}$ R. Breedon, ${ }^{23}$ G. Briskin,${ }^{52}$ R. Brock, ${ }^{43}$ G. Brooijmans, ${ }^{29}$ A. Bross,${ }^{29}$ D. Buchholz, ${ }^{32}$ V. Buescher, ${ }^{47}$ V. S. Burtovoi, ${ }^{19}$ J. M. Butler, ${ }^{40}$ W. Carvalho, ${ }^{3}$ D. Casey, ${ }^{43}$ Z. Casilum, ${ }^{48}$ H. Castilla-Valdez, ${ }^{15}$ D. Chakraborty, ${ }^{48}$ K. M. Chan,${ }^{47}$ S. V. Chekulaev, ${ }^{19}$ W. Chen,${ }^{48}$ D. K. Cho, ${ }^{47}$ S. Choi, ${ }^{26}$ S. Chopra, ${ }^{27}$ B. C. Choudhary, ${ }^{26}$ J. H. Christenson, ${ }^{29}$ M. Chung, ${ }^{30}$ D. Claes, ${ }^{44}$ A. R. Clark, ${ }^{22}$ W. G. Cobau, ${ }^{39}$ J. Cochran, ${ }^{26}$ L. Coney, ${ }^{34}$ B. Connolly, ${ }^{27}$ W. E. Cooper,${ }^{29}$ D. Coppage, ${ }^{36}$ D. Cullen-Vidal, ${ }^{52}$ M. A. C. Cummings, ${ }^{31}$ D. Cutts, ${ }^{52}$ O. I. Dahl, ${ }^{22}$ K. Davis, ${ }^{21}$ K. De, ${ }^{53}$ K. Del Signore, ${ }^{42}$ M. Demarteau,${ }^{29}$ D. Denisov,${ }^{29}$ S. P. Denisov, ${ }^{19}$ H. T. Diehl, ${ }^{29}$ M. Diesburg, ${ }^{29}$ G. Di Loreto, ${ }^{43}$ P. Draper, ${ }^{53}$ Y. Ducros, ${ }^{10}$ L. V. Dudko, ${ }^{18}$ S. R. Dugad,${ }^{13}$ A. Dyshkant, ${ }^{19}$ D. Edmunds, ${ }^{43}$ J. Ellison, ${ }^{26}$ V. D. Elvira ${ }^{29}$ R. Engelmann, ${ }^{48}$ S. Eno, ${ }^{39}$ G. Eppley, ${ }^{55}$ P. Ermolov, ${ }^{18}$

O. V. Eroshin, ${ }^{19}$ J. Estrada, ${ }^{47}$ H. Evans, ${ }^{45}$ V. N. Evdokimov, ${ }^{19}$ T. Fahland,${ }^{25}$ S. Feher, ${ }^{29}$ D. Fein, ${ }^{21}$ T. Ferbel, ${ }^{47}$

H.E. Fisk, ${ }^{29}$ Y. Fisyak,${ }^{49}$ E. Flattum,${ }^{29}$ F. Fleuret,${ }^{22}$ M. Fortner, ${ }^{31}$ K. C. Frame,${ }^{43}$ S. Fuess,${ }^{29}$ E. Gallas, ${ }^{29}$

A. N. Galyaev, ${ }^{19}$ P. Gartung, ${ }^{26}$ V. Gavrilov, ${ }^{17}$ R. J. Genik II ${ }^{20}$ K. Genser ${ }^{29}$ C. E. Gerber, ${ }^{29}$ Y. Gershtein, ${ }^{52}$ B. Gibbard, ${ }^{49}$

R. Gilmartin, ${ }^{27}$ G. Ginther, ${ }^{47}$ B. Gobbi, ${ }^{32}$ B. Gómez, ${ }^{5}$ G. Gómez,${ }^{39}$ P. I. Goncharov, ${ }^{19}$ J. L. González Solís, ${ }^{15}$ H. Gordon, ${ }^{49}$ L. T. Goss, ${ }^{54}$ K. Gounder, ${ }^{26}$ A. Goussiou, ${ }^{48}$ N. Graf, ${ }^{49}$ P. D. Grannis, ${ }^{48}$ J. A. Green, ${ }^{35}$ H. Greenlee ${ }^{29}$

S. Grinstein, ${ }^{1}$ P. Grudberg, ${ }^{22}$ S. Grünendahl, ${ }^{29}$ G. Guglielmo, ${ }^{51}$ A. Gupta, ${ }^{13}$ S. N. Gurzhiev, ${ }^{19}$ G. Gutierrez,${ }^{29}$ P. Gutierrez, ${ }^{51}$ N. J. Hadley, ${ }^{39}$ H. Haggerty, ${ }^{29}$ S. Hagopian, ${ }^{27}$ V. Hagopian, ${ }^{27}$ K. S. Hahn, ${ }^{47}$ R. E. Hall, ${ }^{24}$ P. Hanlet, ${ }^{41}$ S. Hansen, ${ }^{29}$ J. M. Hauptman, ${ }^{35}$ C. Hays,${ }^{45}$ C. Hebert ${ }^{36}$ D. Hedin, ${ }^{31}$ A. P. Heinson, ${ }^{26}$ U. Heintz, ${ }^{40}$ T. Heuring,${ }^{27}$ R. Hirosky, ${ }^{30}$ J. D. Hobbs,${ }^{48}$ B. Hoeneisen, ${ }^{6}$ J. S. Hoftun, ${ }^{52}$ A. S. Ito, ${ }^{29}$ S. A. Jerger ${ }^{43}$ R. Jesik, ${ }^{33}$ T. Joffe-Minor, ${ }^{32}$ K. Johns, ${ }^{21}$ M. Johnson, ${ }^{29}$ A. Jonckheere, ${ }^{29}$ M. Jones, ${ }^{28}$ H. Jöstlein, ${ }^{29}$ S. Y. Jun, ${ }^{32}$ A. Juste, ${ }^{29}$ S. Kahn, ${ }^{49}$ E. Kajfasz, ${ }^{8}$ D. Karmanov, ${ }^{18}$ D. Karmgard, ${ }^{34}$ R. Kehoe, ${ }^{34}$ S. K. Kim,,${ }^{14}$ B. Klima, ${ }^{29}$ C. Klopfenstein, ${ }^{23}$ B. Knuteson, ${ }^{22}$ W. Ko, ${ }^{23}$ J. M. Kohli, ${ }^{11}$ A. V. Kostritskiy, ${ }^{19}$ J. Kotcher, ${ }^{49}$ A. V. Kotwal, ${ }^{45}$ A. V. Kozelov, ${ }^{19}$ E. A. Kozlovsky, ${ }^{19}$ J. Krane, ${ }^{35}$ M. R. Krishnaswamy, ${ }^{13}$ S. Krzywdzinski, ${ }^{29}$ M. Kubantsev, ${ }^{37}$ S. Kuleshov, ${ }^{17}$ Y. Kulik ${ }^{48}$ S. Kunori, ${ }^{39}$ G. Landsberg, ${ }^{52}$ A. Leflat, ${ }^{18}$ F. Lehner, ${ }^{29}$ J. Li ${ }^{53}$ Q. Z. Li, ${ }^{29}$ J. G. R. Lima, ${ }^{3}$ D. Lincoln, ${ }^{29}$ S. L. Linn, ${ }^{27}$ J. Linnemann, ${ }^{43}$ R. Lipton, ${ }^{29}$

J. G. Lu, ${ }^{4}$ A. Lucotte, ${ }^{48}$ L. Lueking, ${ }^{29}$ C. Lundstedt, ${ }^{44}$ A. K. A. Maciel, ${ }^{31}$ R. J. Madaras,${ }^{22}$ V. Manankov,${ }^{18}$

S. Mani, ${ }^{23}$ H. S. Mao, ${ }^{4}$ R. Markeloff, ${ }^{31}$ T. Marshall, ${ }^{33}$ M. I. Martin, ${ }^{29}$ R. D. Martin,${ }^{30}$ K. M. Mauritz,${ }^{35}$ B. May, ${ }^{32}$ A. A. Mayorov, ${ }^{33}$ R. McCarthy, ${ }^{48}$ J. McDonald, ${ }^{27}$ T. McKibben, ${ }^{30}$ T. McMahon, ${ }^{50}$ H. L. Melanson, ${ }^{29}$ M. Merkin, ${ }^{18}$ K. W. Merritt, ${ }^{29}$ C. Miao,${ }^{52}$ H. Miettinen, ${ }^{55}$ D. Mihalcea, ${ }^{51}$ A. Mincer ${ }^{46}$ C. S. Mishra, ${ }^{29}$ N. Mokhov, ${ }^{29}$ N. K. Mondal, ${ }^{13}$

H.E. Montgomery, ${ }^{29}$ M. Mostafa, ${ }^{1}$ H. da Motta, ${ }^{2}$ E. Nagy, ${ }^{8}$ F. Nang, ${ }^{21}$ M. Narain,${ }^{40}$ V. S. Narasimham,${ }^{13}$

H. A. Neal, ${ }^{42}$ J. P. Negret,${ }^{5}$ S. Negroni,${ }^{8}$ D. Norman, ${ }^{54}$ L. Oesch,${ }^{42}$ V. Oguri, ${ }^{3}$ B. Olivier, ${ }^{9}$ N. Oshima,${ }^{29}$ P. Padley, ${ }^{55}$ L. J. Pan ${ }^{32}$ A. Para,${ }^{29}$ N. Parashar, ${ }^{41}$ R. Partridge,${ }^{52}$ N. Parua,${ }^{7}$ M. Paterno, ${ }^{47}$ A. Patwa, ${ }^{48}$ B. Pawlik, ${ }^{16}$ J. Perkins, ${ }^{53}$ M. Peters ${ }^{28}$ R. Piegaia, ${ }^{1}$ H. Piekarz,${ }^{27}$ B. G. Pope,${ }^{43}$ E. Popkov, ${ }^{34}$ H. B. Prosper,${ }^{27}$ S. Protopopescu, ${ }^{49}$ J. Qian, ${ }^{42}$ P.Z. Quintas, ${ }^{29}$ R. Raja, ${ }^{29}$ S. Rajagopalan, ${ }^{49}$ N. W. Reay, ${ }^{37}$ S. Reucroft,${ }^{41}$ M. Rijssenbeek, ${ }^{48}$ T. Rockwell, ${ }^{43}$ M. Roco, ${ }^{29}$ P. Rubinov, ${ }^{32}$ R. Ruchti, ${ }^{34}$ J. Rutherfoord, ${ }^{21}$ A. Santoro, ${ }^{2}$ L. Sawyer, ${ }^{38}$ R. D. Schamberger, ${ }^{48}$ H. Schellman, ${ }^{32}$

A. Schwartzman, ${ }^{1}$ J. Sculli, ${ }^{46}$ N. Sen, ${ }^{55}$ E. Shabalina, ${ }^{18}$ H. C. Shankar, ${ }^{13}$ R. K. Shivpuri, ${ }^{12}$ D. Shpakov, ${ }^{48}$ M. Shupe,${ }^{21}$ R. A. Sidwell, ${ }^{37}$ H. Singh, ${ }^{26}$ J. B. Singh, ${ }^{11}$ V. Sirotenko, ${ }^{31}$ P. Slattery, ${ }^{47}$ E. Smith, ${ }^{51}$ R. P. Smith, ${ }^{29}$ R. Snihur,${ }^{32}$ G. R. Snow, ${ }^{44}$ J. Snow, ${ }^{50}$ S. Snyder, ${ }^{49}$ J. Solomon, ${ }^{30}$ X. F. Song, ${ }^{4}$ V. Sorín, ${ }^{1}$ M. Sosebee,${ }^{53}$ N. Sotnikova, ${ }^{18}$ M.Souza, ${ }^{2}$ N. R. Stanton, ${ }^{37}$ G. Steinbrück, ${ }^{45}$ R. W. Stephens, ${ }^{53}$ M. L. Stevenson, ${ }^{22}$ F. Stichelbaut, ${ }^{49}$ D. Stoker, ${ }^{25}$ V. Stolin, ${ }^{17}$ D. A. Stoyanova, ${ }^{19}$ M. Strauss, ${ }^{51}$ K. Streets, ${ }^{46}$ M. Strovink,${ }^{22}$ L. Stutte, ${ }^{29}$ A. Sznajder, ${ }^{3}$ J. Tarazi,${ }^{25}$ W. Taylor ${ }^{48}$ S. Tentindo-Repond, ${ }^{27}$ T. L. T. Thomas, ${ }^{32}$ J. Thompson, ${ }^{39}$ D. Toback, ${ }^{39}$ T. G. Trippe, ${ }^{22}$ A. S. Turcot, ${ }^{42}$ P. M. Tuts, ${ }^{45}$ P. van Gemmeren, ${ }^{29}$ V. Vaniev, ${ }^{19}$ N. Varelas,${ }^{30}$ A. A. Volkov, ${ }^{19}$ A. P. Vorobiev, ${ }^{19}$ H. D. Wahl, ${ }^{27}$ H. Wang, ${ }^{32}$ J. Warchol, ${ }^{34}$ G. Watts, ${ }^{56}$ M. Wayne, ${ }^{34}$ H. Weerts, ${ }^{43}$ A. White, ${ }^{53}$ J. T. White, ${ }^{54}$ D. Whiteson, ${ }^{22}$ J. A. Wightman, ${ }^{35}$ S. Willis, ${ }^{31}$ S. J. Wimpenny, ${ }^{26}$ J. V. D. Wirjawan, ${ }^{54}$ J. Womersley, ${ }^{29}$ D. R. Wood, ${ }^{41}$ R. Yamada,${ }^{29}$ P. Yamin, ${ }^{49}$ T. Yasuda, ${ }^{29}$ K. Yip, ${ }^{29}$ S. Youssef, ${ }^{27}$ J. Yu, ${ }^{29}$ Z. Yu, ${ }^{32}$ M. Zanabria, ${ }^{5}$ H. Zheng, ${ }^{34}$ Z. Zhou,${ }^{35}$ Z. H. Zhu, ${ }^{47}$ M. Zielinski, ${ }^{47}$ D. Zieminska, ${ }^{33}$ A. Zieminski, ${ }^{33}$ V. Zutshi, ${ }^{47}$ E. G. Zverev, ${ }^{18}$ and A. Zylberstejn ${ }^{10}$ 


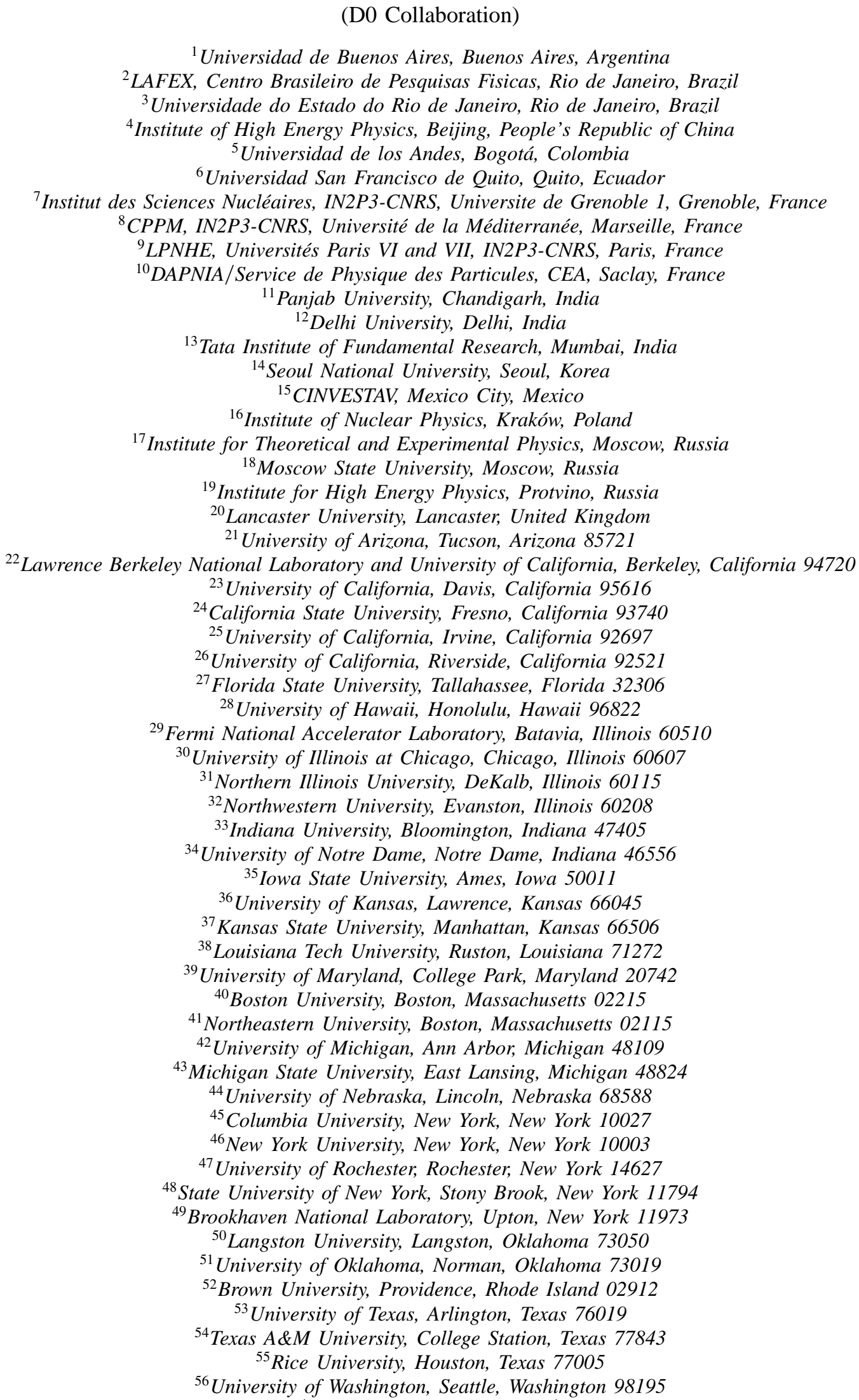

Inclusive dijet production at large pseudorapidity intervals $(\Delta \eta)$ between the two jets has been suggested as a regime for observing Balitsky-Fadin-Kuraev-Lipatov (BFKL) dynamics. We have measured the dijet cross section for large $\Delta \eta$ in $p \bar{p}$ collisions at $\sqrt{s}=1800$ and $630 \mathrm{GeV}$ using the D0 
detector. The partonic cross section increases strongly with the size of $\Delta \eta$. The observed growth is even stronger than expected on the basis of BFKL resummation in the leading logarithmic approximation. The growth of the partonic cross section can be accommodated with an effective BFKL intercept of $\alpha_{\mathrm{BFKL}}(20 \mathrm{GeV})=1.65 \pm 0.07$.

PACS numbers: 13.87. $-\mathrm{a}, 12.38 .-\mathrm{t}$

Jet production in the high-energy limit of quantum chromodynamics (QCD), as defined by center-of-mass (c.m.) energies $(\sqrt{s})$ much larger than the momentum transfers $(Q)$, presents a very interesting and yet little explored area. In this kinematic region, the significantly different energy scales of the process lead to calculated jet cross sections characterized by the appearance of large logarithms $\ln \left(s / Q^{2}\right)$, which must be summed to all orders in $\alpha_{s}$. This summation is accomplished through the Balitsky-Fadin-Kuraev-Lipatov (BFKL) [1] equation, which involves a spacelike chain of an infinite number of gluon emissions. The gluons have similar transverse momenta, but they are strongly ordered in their pseudorapidities or, equivalently, in their longitudinal momentum fractions $x_{i}$. Thus, the BFKL equation effectively describes the evolution in $x$ (growth with $1 / x$ ) of the gluon momentum distribution in the proton.

Attempts to isolate and probe the BFKL evolution in the low- $x$ region in $e p$ collisions at HERA, by measuring the forward jet and particle cross sections [2], have led to ambiguous results. (At HERA, forward denotes the region away from the current jet and towards the proton remnant.) In $p \bar{p}$ collisions, the azimuthal decorrelation as a function of the pseudorapidity interval, $\Delta \eta$, in dijet systems has been studied [3]. (Here, $\eta=-\ln [\tan (\theta / 2)]$, where $\theta$ is the polar angle of the jet relative to the proton beam.) It has been argued [4], however, that the azimuthal angle distribution is not an inclusive enough quantity for the cancellation between the real and virtual soft gluons that accompany dijet production; thus, such a distribution cannot be used as a probe of the BFKL equation.

Inclusive dijet production at large pseudorapidity intervals in high-energy $p \bar{p}$ collisions, on the other hand, provides an excellent testing ground for BFKL dynamics. We present a measurement of the dijet cross section at large $\Delta \eta$ using the D0 detector at the Fermilab Tevatron collider. We reconstruct the event kinematics using the most forward/backward jets, and measure the cross section as a function of $x_{1}, x_{2}$, and $Q^{2}$. The longitudinal momentum fractions of the proton and antiproton, $x_{1}$ and $x_{2}$, carried by the two interacting partons are defined as

$$
x_{1,2}=\frac{2 E_{T_{1,2}}}{\sqrt{s}} e^{ \pm \bar{\eta}} \cosh (\Delta \eta / 2),
$$

where $E_{T_{1}}\left(E_{T_{2}}\right)$ and $\eta_{1}\left(\eta_{2}\right)$ are the transverse energy and pseudorapidity of the most forward(backward) jet, $\Delta \eta=$ $\eta_{1}-\eta_{2} \geq 0$, and $\bar{\eta}=\left(\eta_{1}+\eta_{2}\right) / 2$. The momentum transfer during the hard scattering is defined as

$$
Q=\sqrt{E_{T_{1}} E_{T_{2}}} \text {. }
$$

The total dijet cross section, $\sigma$, can be factorized into the partonic cross section, $\hat{\sigma}$, and the parton distribution functions (PDF), $P\left(x_{1,2}, Q^{2}\right)$, in the proton and antiproton: $\sigma=x_{1} P\left(x_{1}, Q^{2}\right) x_{2} P\left(x_{2}, Q^{2}\right) \hat{\sigma}$. The partonic c.m. energy, $\sqrt{\hat{s}}$, equals $\sqrt{x_{1} x_{2} s}$. For sufficiently large values of $x_{1}$ and $x_{2}$, any large $\alpha_{s} \ln \left(s / Q^{2}\right)$ terms in $\sigma$ correspond to large $\alpha_{s} \ln \left(\hat{s} / Q^{2}\right)$, which are of the order of $\alpha_{s} \Delta \eta$, and factorize in $\hat{\sigma}$. Using the BFKL prescription to sum the leading logarithmic terms $\alpha_{s} \ln \left(\hat{s} / Q^{2}\right)$ to all orders in $\alpha_{s}$, results in an exponential rise of $\hat{\sigma}$ with $\Delta \eta$ [5]:

$$
\hat{\sigma}_{\mathrm{BFKL}} \propto \frac{1}{Q^{2}} \cdot \frac{e^{\left(\alpha_{\mathrm{BFKL}}-1\right) \Delta \eta}}{\sqrt{\alpha_{s} \Delta \eta}},
$$

where $\alpha_{\mathrm{BFKL}}$ is the BFKL intercept that governs the strength of the growth of the gluon distribution at small $x$. In the leading logarithmic approximation (LLA), $\alpha_{\mathrm{BFKL}}$ is given by [1]:

$$
\alpha_{\mathrm{BFKL}}-1=\frac{\alpha_{s}(Q) 12 \ln 2}{\pi} .
$$

The predicted rise of the partonic cross section with $\Delta \eta$ is difficult to observe experimentally due to the dependence of the total cross section on the PDF. To overcome this difficulty, we measure the cross section at two c.m. energies, $\sqrt{s_{A}}=1800 \mathrm{GeV}$ and $\sqrt{s_{B}}=630 \mathrm{GeV}$, and take their ratio for the same values of $x_{1}, x_{2}$, and $Q^{2}$. This eliminates the dependence on the PDF, and reduces the ratio to that of the partonic cross sections. The latter is purely a function of the $\Delta \eta$ values:

$$
R \equiv \frac{\sigma\left(\sqrt{s_{A}}\right)}{\sigma\left(\sqrt{s_{B}}\right)}=\frac{\hat{\sigma}\left(\Delta \eta_{A}\right)}{\hat{\sigma}\left(\Delta \eta_{B}\right)}=\frac{e^{\left(\alpha_{\mathrm{BFKL}}-1\right)\left(\Delta \eta_{A}-\Delta \eta_{B}\right)}}{\sqrt{\Delta \eta_{A} / \Delta \eta_{B}}} .
$$

Thus, varying $\sqrt{s}$, while keeping $x_{1}, x_{2}$, and $Q^{2}$ fixed, is equivalent to varying $\Delta \eta$, which directly probes the BFKL dynamics. In addition, measurement of the ratio leads to cancellation of certain experimental uncertainties, and enables an experimental extraction of $\alpha_{\mathrm{BFKL}}$.

In the D0 [6] detector, jets are identified using the uranium/liquid-argon calorimeters. These cover the range of $|\eta| \leq 4.1$, and are segmented into towers of $\Delta \eta \times \Delta \phi=$ $0.1 \times 0.1(\phi$ is the azimuthal angle $)$.

The data samples for this analysis were collected during the 1995-1996 Tevatron Collider run. Events were selected online by a three-level trigger system culminating in the software trigger requirement of a jet candidate with $E_{T}>12 \mathrm{GeV}$. The trigger was $85 \%$ efficient for jets with $E_{T}=20 \mathrm{GeV}$, and fully efficient for jets with $E_{T}>30 \mathrm{GeV}$. The integrated luminosity of the trigger was $0.7 \mathrm{nb}^{-1}$ for the $\sqrt{s}=1800 \mathrm{GeV}$ sample, and $31.8 \mathrm{nb}^{-1}$ for the $\sqrt{s}=630 \mathrm{GeV}$ sample [7]. 
Jets were reconstructed offline using an iterative fixedcone algorithm with a cone radius of $\mathcal{R}=0.7$ in $(\eta, \phi)$ space [8]. The pseudorapidity of each jet was corrected for small reconstruction and jet algorithm biases. The transverse energy of each jet was corrected in three stages: (i) Energy originating from spectator parton interactions, additional $p \bar{p}$ interactions, noise from uranium decay, and residual energy from previous $p \bar{p}$ interactions was subtracted on average from the measured jet energy [9]; (ii) The jet energy was corrected for the hadronic response of the calorimeter [9]; (iii) The fraction of the particle energy that showered outside of the jet reconstruction cone was recovered, and the fraction of the energy reconstructed within the cone that did not belong to the original particle was subtracted [10]. The average correction for jets of $E_{T}=20 \mathrm{GeV}$ and $|\eta|=2.5$ is $(22.8 \pm 4.8) \%$ at $\sqrt{s}=$ $1800 \mathrm{GeV}$; for jets of the same $E_{T}$ and $|\eta|=1.2$ the correction is $(14.5 \pm 4.0) \%$ at $630 \mathrm{GeV}$.

The event vertex was required to lie within $50 \mathrm{~cm}$ of the detector center; $93 \%(86) \%$ of the events at $1800(630) \mathrm{GeV}$ satisfied this requirement. To remove cosmic ray background, the imbalance in the transverse momentum of the event was required to be less than $70 \%$ of the leading jet $E_{T}$; more than $98 \%$ of the events at each c.m. energy satisfied this requirement. To ensure good jet reconstruction efficiency and jet energy calibration, jets were selected with $E_{T}>20 \mathrm{GeV}$ and $|\eta|<3$. Backgrounds from isolated noisy calorimeter cells, accelerator beam losses, and electromagnetic clusters that mimic jets were eliminated by applying a series of jet quality criteria; $97 \%$ of the jets survived this final selection.

The selected jets of each event were ordered in pseudorapidity. A minimum pseudorapidity interval of $\Delta \eta>2$ was required between the most forward and most backward jet. In the final samples, the most forward and most backward jets were found to have approximately the same $E_{T}$. The values of $x_{1}, x_{2}$, and $Q^{2}$ were calculated from Eqs. (1) and (2). Most of the data at $\sqrt{s}=1800 \mathrm{GeV}$ are within $0.01<x_{1,2}<0.30$, and at $630 \mathrm{GeV}$, within $0.03<x_{1,2}<0.60$. The region of maximum overlap, $0.06<x_{1,2}<0.30$, was divided into six equal bins of $x_{1}$ and $x_{2}$. Because of limited statistics, only one bin in $Q^{2}$ was used: $400<Q^{2}<1000 \mathrm{GeV}^{2}$. The dijet cross section, corrected for trigger, event, and jet selection inefficiencies, was computed in each $\left(x_{1}, x_{2}, Q^{2}\right)$ bin.

The dijet cross section at low $\left(x_{1}, x_{2}\right)$ is affected by the acceptance of the $E_{T}>20 \mathrm{GeV}$ and $\Delta \eta>2$ requirements. To avoid this bias, we require $x_{1} \cdot x_{2}>0.01$. Similarly, the cross section at high $\left(x_{1}, x_{2}\right)$ is biased by the $|\eta|<3$ requirement, so that we require $x_{1,2}<0.22$. A total of ten $\left(x_{1}, x_{2}\right)$ bins satisfy both requirements.

Multiple $p \bar{p}$ interactions during the same beam crossing, which, in principle, could distort the topology of the event and bias the cross section, were infrequent for the low instantaneous luminosity $\left[\mathcal{L}<10^{30}(2 \times\right.$ $\left.10^{30}\right) \mathrm{cm}^{-2} \mathrm{~s}^{-1}$ at $\left.\sqrt{s}=1800(630) \mathrm{GeV}\right]$ data used in this analysis. Nevertheless, any possible luminosity effects on the dijet cross section were evaluated by measuring the cross section at $630 \mathrm{GeV}$ from lower- and higher-luminosity subsamples. No significant difference was observed between the two measurements.

The dijet cross section is distorted by jet energy resolution. The resolution was measured as a function of jet pseudorapidity and $E_{T}$, by balancing $E_{T}$ in events with only two jets back-to-back in $\phi$. For jets of $E_{T}=20 \mathrm{GeV}$, the fractional $E_{T}$ resolution is $27 \%(14 \%)$ at $|\eta|=1.2(2.5)$ and $\sqrt{s}=1800 \mathrm{GeV}$ (measured from the jet data collected during the 1994-1995 $92 \mathrm{pb}^{-1}$ Tevatron run). At $\sqrt{s}=630 \mathrm{GeV}$, limited statistics prohibited the measurement of the resolutions in the whole $E_{T}$ and $\eta$ spectrum. In the regions where the measurement was possible, the resolutions at $630 \mathrm{GeV}$ were found to be smaller than the resolutions at $1800 \mathrm{GeV}$ by $\sim 1 \%$.

The distortion of the cross section was corrected using the HERWIG [11] Monte Carlo (MC) event generator, convoluted with the CTEQ4M [12] PDF. In the MC events, the jet transverse energies were smeared using the resolutions extracted from the $1800 \mathrm{GeV}$ data. The $E_{T}>20 \mathrm{GeV}$, $|\eta|<3$, and $\Delta \eta>2$ requirements were applied separately to the original fully fragmented (particle-level) jets and to the $E_{T}$-smeared jets. Particle-level and smeared dijet cross sections were calculated in the same $\left(x_{1}, x_{2}, Q^{2}\right)$ bins as in the data. Apart from normalization differences, the smeared HERWIG cross section at both c.m. energies exhibits the same dependence on $x_{1,2}$ as the data. The ratio of the particle-level to the smeared MC cross section in each bin was used as an unsmearing factor to correct the data cross section for the jet energy resolution effects. The unsmearing correction for the dijet cross section is typically of the order of $10 \%$ at both c.m. energies, whereas the unsmearing correction for the ratio of the cross sections amounts to only $6 \%$. The difference between the measured resolutions at the two c.m. energies was accounted for in the systematic uncertainties. The unsmearing method was verified by using a smeared MC sample generated with ISAJET [13], and comparing the ISAJET particle-level cross section to that obtained using our unsmearing procedure based on HERWIG.

The dijet cross sections for $\Delta \eta>2$ at $\sqrt{s}=1800$ and $630 \mathrm{GeV}$ in the selected $\left(x_{1}, x_{2}\right)$ bins are shown in Table I. In each bin, the average values of $x_{1}, x_{2}$, and $Q^{2}$ are in good agreement, within the precision of our measurement, between the two c.m. energies. This ensures the cancellation of the PDF in the ratio of the cross sections. Also shown in the Table are the values for the BFKL intercept, $\alpha_{\mathrm{BFKL}}$, extracted from the cross sections and the average pseudorapidity intervals at 1800 and $630 \mathrm{GeV}$ in each $\left(x_{1}, x_{2}\right)$ bin, using Eq. (5).

The mean value of the ratios of the cross sections in the ten bins is equal to $\langle R\rangle \equiv\left\langle\sigma_{1800} / \sigma_{630}\right\rangle=2.8 \pm 0.3$ (stat). The mean value of $\alpha_{\mathrm{BFKL}}$ is equal to $1.65 \pm 0.05$ (stat). The mean pseudorapidity interval, $\langle\Delta \eta\rangle$, in the selected bins is equal to 4.6 units at $1800 \mathrm{GeV}$ and 2.4 units at $630 \mathrm{GeV}$. 
TABLE I. The dijet cross sections for $\Delta \eta>2$ at $\sqrt{s}=1800$ and $630 \mathrm{GeV}$ and the extracted value of the BFKL intercept in each of the ten $\left(x_{1}, x_{2}\right)$ bins. The minimum jet $E_{T}$ is $20 \mathrm{GeV}$. The uncertainties are statistical.

\begin{tabular}{|c|c|c|c|c|}
\hline$x_{1}$ range & $x_{2}$ range & $\begin{array}{c}\sigma_{1800} \\
(\mathrm{nb})\end{array}$ & $\begin{array}{l}\sigma_{630} \\
(\mathrm{nb})\end{array}$ & $\alpha_{\mathrm{BFKL}}$ \\
\hline $0.06-0.10$ & $0.18-0.22$ & $28.1 \pm 6.9$ & $8.4 \pm 0.9$ & $1.74 \pm 0.13$ \\
\hline 14 & $\begin{array}{l}0.14-0.18 \\
0.18-0.22\end{array}$ & $\begin{array}{r}40.1 \pm 9.5 \\
3.6 \pm 2.1\end{array}$ & $\begin{array}{l}8.8 \pm 0.9 \\
5.4 \pm 0.6\end{array}$ & $\begin{array}{l}1.83 \pm 0.11 \\
0.96 \pm 0.49 \\
-0.28\end{array}$ \\
\hline $\begin{array}{c}0.14-0.18 \\
\ldots \\
\ldots\end{array}$ & $\begin{array}{l}0.10-0.14 \\
0.14-0.18 \\
0.18-0.22\end{array}$ & $\begin{array}{r}27.9 \pm 7.3 \\
10.4 \pm 6.1 \\
5.6 \pm 4.5 \\
-3.8\end{array}$ & $\begin{array}{l}8.4 \pm 0.8 \\
5.0 \pm 0.6 \\
2.9 \pm 0.5\end{array}$ & $\begin{array}{l}1.71 \pm 0.13 \\
1.50 \pm 0.29 \\
1.44 \pm 0.38 \\
-0.32\end{array}$ \\
\hline $\begin{array}{c}0.18-0.22 \\
\ldots \\
\ldots \\
\ldots\end{array}$ & $\begin{array}{l}0.06-0.10 \\
0.10-0.14 \\
0.14-0.18 \\
0.18-0.22\end{array}$ & $\begin{array}{r}26.3 \pm 6.6 \\
12.5 \pm 6.3 \\
6.8 \pm 5.0 \\
2.4 \pm 1.7\end{array}$ & $\begin{array}{l}8.6 \pm 0.9 \\
6.3 \pm 0.7 \\
3.1 \pm 0.4 \\
1.7 \pm 0.3\end{array}$ & $\begin{array}{l}1.71 \pm 0.14 \\
1.46 \pm 0.24 \\
1.50 \pm 0.24 \\
1.28 \pm 0.60 \\
-0.37\end{array}$ \\
\hline
\end{tabular}

The largest sources of systematic uncertainties on the ratio of the cross sections and the BFKL intercept are the jet energy scale (yielding an $8 \%$ uncertainty on the ratio and $2 \%$ on the intercept) and the jet energy resolutions (7\% on the ratio and $2 \%$ on the intercept). The individual components of these were evaluated for correlations between the two data samples. Additional sources of systematic uncertainties on the ratio and the intercept include the choice of the input PDF in the Monte Carlo used for unsmearing ( $1 \%$ on the ratio, negligible on the intercept), and the uncertainty in the normalization of the luminosity ( $2 \%$ on the ratio and $1 \%$ on the intercept). The total systematic uncertainty amounts to $11 \%$ on the ratio of the cross sections and 3\% on the BFKL intercept, yielding the final results:

$$
\begin{aligned}
&\langle R\rangle=2.8 \pm 0.3 \text { (stat) } \pm 0.3 \text { (syst) }=2.8 \pm 0.4 \\
&\left\langle\alpha_{\mathrm{BFKL}}\right\rangle=1.65 \pm 0.05(\text { stat }) \pm 0.05 \text { (syst) } \\
&=1.65 \pm 0.07 .
\end{aligned}
$$

Hence, for the same values of $x_{1}, x_{2}$, and $Q^{2}$, the dijet cross section at large $\Delta \eta$ increases by almost a factor of 3 between the two c.m. energies, corresponding to the increase of $\langle\Delta \eta\rangle$ from 2.4 to 4.6 units.

Several theoretical predictions can be compared to our measurement. Leading order QCD predicts the ratio of the cross sections to fall asymptotically toward unity with increasing $\Delta \eta$. For the $\Delta \eta$ values relevant to this analysis, the predicted ratio is $R_{\mathrm{LO}}=1.2$ [14].

The HERWIG MC provides an alternative prediction. It calculates the exact $2 \rightarrow 2$ subprocess, including initial and final state radiation and angular ordering of the emitted partons. Using the same $\left(x_{1}, x_{2}, Q^{2}\right)$ bins as in the data yields $R_{\text {HER WIG }}=1.6 \pm 0.1$ (stat).

The LLA BFKL intercept according to Eq. (4) for $\alpha_{s}(20 \mathrm{GeV})=0.17 \quad[14]$ is $\alpha_{\mathrm{BFKL}, \mathrm{LLA}}=1.45$. For $\Delta \eta_{1800}=4.6$ and $\Delta \eta_{630}=2.4$, Eq. (5) yields $R_{\mathrm{BFKL}, \mathrm{LLA}}=1.9$. It should be noted, however, that the leading log approximation may be too simplistic, and that exact quantitative predictions including the next-toleading logarithmic [15] corrections to the BFKL kernel are not as yet available.

It is evident that the growth of the dijet cross section with $\Delta \eta$ (from $\langle\Delta \eta\rangle=2.4$ to 4.6 ) is stronger in the data than in the theoretical models we considered. The measured ratio is higher as listed: by 4 standard deviations than the LO prediction, by 3 deviations than the HERWIG prediction, and by 2.3 deviations than the LLA BFKL prediction.

It should be noted that the $x_{1,2}$ definitions of Eq. (1) have been kept the same in the data and in the theoretical calculations. Modifying these definitions to account for all jets in the event changes the ratio of the cross sections by less than $10 \%$.

Finally, the $\Delta \eta>2$ requirement was changed to $\Delta \eta>$ 1 , and the analysis was repeated. For $\Delta \eta>1$, Eq. (1) yields $x_{1} \cdot x_{2}>0.005$, which results in a selection of fifteen unbiased $\left(x_{1}, x_{2}\right)$ bins. The mean pseudorapidity interval in the selected bins is equal to 4.2 at $1800 \mathrm{GeV}$ and 1.9 at $630 \mathrm{GeV}$. The average ratio of the 1800 and $630 \mathrm{GeV}$ cross sections in the selected bins was measured to be $1.8 \pm 0.1$ (stat) \pm 0.1 (uncorrelated syst). The results are shown in Fig. 1 as a function of the mean pseudorapidity interval at $\sqrt{s}=630 \mathrm{GeV}$. In the case of the $\Delta \eta>1$ requirement, the observed ratio is once again larger than the exact LO and HERWIG predictions. It is interesting, however, that HERWIG exhibits the same qualitative behavior as the data in that the ratio of cross sections decreases as the $\Delta \eta$ requirement is relaxed, whereas the exact LO

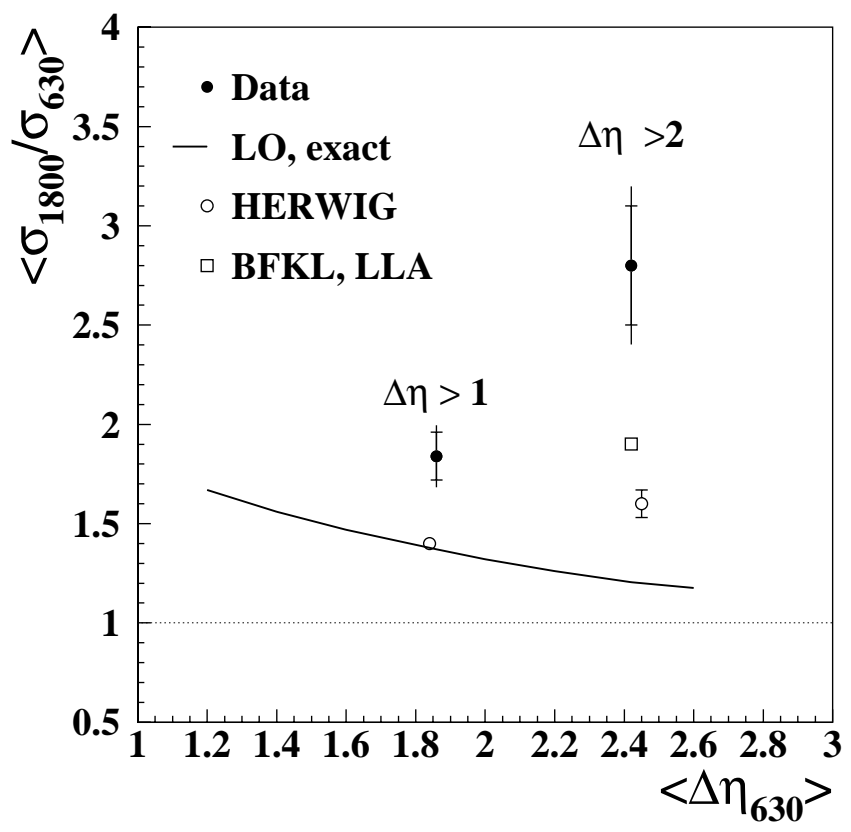

FIG. 1. The ratio of the dijet cross sections at $\sqrt{s}=1800$ and $630 \mathrm{GeV}$ for $\Delta \eta>1$ and $\Delta \eta>2$. The minimum jet $E_{T}$ is $20 \mathrm{GeV}$. The inner error bars on the data points represent statistical uncertainties; the outer bars represent statistical and uncorrelated systematic uncertainties added in quadrature. The error bars on the HERWIG predictions represent statistical uncertainties. The $\mathrm{LO}$ and BFKL predictions are analytical calculations. 
calculation predicts a very different trend. (A BFKL prediction is not shown for the case of $\Delta \eta>1$ since the pseudorapidity interval is not sufficiently large for the formalism to be meaningful.)

In conclusion, we have measured the dijet cross section for large pseudorapidity intervals at $\sqrt{s}=1800$ and $630 \mathrm{GeV}$, and the ratio of the cross sections for the same values of $x_{1}, x_{2}$, and $Q^{2}$ at the two energies. The latter corresponds to the ratio of the partonic cross sections for different values of $\Delta \eta$. The measured partonic cross section increases strongly with $\Delta \eta$, more strongly than expected on the basis of any current prediction.

We appreciate the many fruitful discussions with A. Mueller, L. Orr, and J. Stirling. We thank the staffs at Fermilab and at collaborating institutions for contributions to this work, and acknowledge support from the Department of Energy and National Science Foundation (U.S.A.), Commissariat à L'Energie Atomique and CNRS/Institut National de Physique Nucléaire et de Physique des Particules (France), Ministry for Science and Technology and Ministry for Atomic Energy (Russia), CAPES and CNPq (Brazil), Departments of Atomic Energy and Science and Education (India), Colciencias (Colombia), CONACyT (Mexico), Ministry of Education and KOSEF (Korea), CONICET and UBACyT (Argentina), A. P. Sloan Foundation, and the Humboldt Foundation.

[1] L. N. Lipatov, Sov. J. Nucl. Phys. 23, 338 (1976); E. A. Kuraev, L. N. Lipatov, and V. S. Fadin, Sov. Phys. JETP 44,
443 (1976); Sov. Phys. JETP 45, 199 (1977); Y. Y. Balitsky and L. N. Lipatov, Sov. J. Nucl. Phys. 28, 822 (1978).

[2] H1 Collaboration, S. Aid et al., Phys. Lett. B 356, 118 (1995); H1 Collaboration, C. Adloff et al., Nucl. Phys. B538, 3 (1999); H1 Collaboration, C. Adloff et al., Phys. Lett. B 462, 440 (1999); ZEUS Collaboration, J. Breitweg et al., Eur. Phys. J. C6, 239 (1999); ZEUS Collaboration, J. Breitweg et al., Phys. Lett. B 474, 223 (2000).

[3] D0 Collaboration, B. Abbott et al., Phys. Rev. Lett. 77, 595 (1996).

[4] Y.L. Dokshitzer, in Proceedings of the 1997 International Europhysics Conference on High Energy Physics, Jerusalem, Israel, 1997 (Springer, New York, 1999), p. 47.

[5] A. H. Mueller and H. Navelet, Nucl. Phys. B282, 727 (1987).

[6] D0 Collaboration, S. Abachi et al., Nucl. Instrum. Methods Phys. Res., Sect. A 338, 185 (1994).

[7] J. Bantly et al., Report No. Fermilab-TM-1995, 1997; J. Krane, J. Bantly, and D. Owen, Report No. Fermilab-TM2000, 1997.

[8] B. Abbott et al., Report No. Fermilab-Pub-97/242-E, 1997.

[9] D0 Collaboration, B. Abbott et al., Nucl. Instrum. Methods Phys. Res., Sect. A 424, 352 (1999).

[10] A. Goussiou, Report No. Fermilab-Pub-99/264-E, 1999.

[11] G. Marchesini et al., Comput. Phys. Commun. 67, 465 (1992). We used v5.9.

[12] H. L. Lai et al., Phys. Rev. D 55, 1280 (1997).

[13] F. E. Paige et al., Report No. BNL-HET-98-39, 1998.

[14] L. H. Orr and W. J. Stirling, Phys. Lett. B 429, 135 (1998).

[15] V.S. Fadin and L. N. Lipatov, Phys. Lett. B 429, 127 (1998); G. Camici and M. Ciafaloni, Phys. Lett. B 430, 349 (1998). 